\title{
SPIN-ORBIT CORRELATIONS AND SINGLE-SPIN ASYMMETRIES
}

\author{
M. BURKARDT \\ Dept. of Physics, New Mexico State University, Las Cruces, NM 88003, USA \\ E-mail: burkardt@nmsu.edu*
}

\begin{abstract}
Several examples for the role of orbital angular momentum and spin-orbit correla-
\end{abstract} tions in hadron structure are discussed.

\section{Introduction}

In nonrelativistic quantum mechanics, Fourier transforms of the form factors yield charge distributions in the center of mass frame. In general, the concept of a center of mass has no analog in relativistic theories, and thus the position space interpretation of form factors is frame dependent. This is different in the Infinite Momentum Frame (IMF) or light-cone framework, where a Galilean subgroup of transverse boosts allows introducing the transverse center of longitudinal momentum as the weighted average of transverse (i.e. $\perp$ to the boost direction) positions of all partons, weighted by their momentum fractions. This center of $\perp$ momentum is the reference point in the interpretation of the (two dimensional) Fourier transform of Generalized Parton Distributions (GPDs) as Impact Parameter dependent parton Distributions (IPDs) [1]. A similar interpretation exists for the 2-d Fourier transform of form factors [2] as the latter can also be obtained by integrating GPDs over the momentum fraction.

The distribution of partons in impact parameter also plays a role for SingleSpin Asymmetries (SSAs): as one expects the final state interactions on the ejected quark in a Semi-Inclusive Deep-Inelastic Scattering (SIDIS) experiment to be on average attractive, any sideward deformation of IPDs is expected to result in an enhancement of the transverse momentum distribution of the ejected quark in the opposite direction. This observation forms the basis for a qualitative link between GPDs and SSAs [3].

*this work was supported by the doe (DE-FG03-95ER40965) 


\section{Impact Parameter Dependent PDFs and the Sivers Effect}

The Fourier transform of the GPD $H_{q}(x, 0, t)$ yields the distribution $q\left(x, \mathbf{b}_{\perp}\right)$ of unpolarized quarks, for an unpolarized target, in impact parameter space

$$
q\left(x, \mathbf{b}_{\perp}\right)=\int \frac{d^{2} \boldsymbol{\Delta}_{\perp}}{(2 \pi)^{2}} H_{q}\left(x, 0,-\boldsymbol{\Delta}_{\perp}^{2}\right) e^{-i \mathbf{b}_{\perp} \cdot \boldsymbol{\Delta}_{\perp}},
$$

with $\boldsymbol{\Delta}_{\perp}=\mathbf{p}_{\perp}^{\prime}-\mathbf{p}_{\perp}$. For a transversely polarized target (e.g. when polarized in the $+\hat{x}$-direction) the impact parameter dependent PDF (IPD) $q_{+\hat{x}}\left(x, \mathbf{b}_{\perp}\right)$ is no longer axially symmetric and the transverse deformation is described by the gradient of the Fourier transform of the GPD $E_{q}(x, 0, t)$

$$
q_{+\hat{x}}\left(x, \mathbf{b}_{\perp}\right)=q\left(x, \mathbf{b}_{\perp}\right)-\frac{1}{2 M} \frac{\partial}{\partial b_{y}} \int \frac{d^{2} \boldsymbol{\Delta}_{\perp}}{(2 \pi)^{2}} E_{q}\left(x, 0,-\boldsymbol{\Delta}_{\perp}^{2}\right) e^{-i \mathbf{b}_{\perp} \cdot \boldsymbol{\Delta}_{\perp}}
$$

$E_{q}(x, 0, t)$ and hence the details of this deformation are not very well known, but its $x$-integral, the Pauli form factor $F_{2}$, is. This allows to relate the average transverse deformation resulting from Eq. (2) to the contribution from the corresponding quark flavor to the anomalous magnetic moment. This observation is important in understanding the sign of the Sivers function.

In a target that is polarized transversely (e.g. vertically), the quarks in the target nucleon can exhibit a (left/right) asymmetry of the distribution $f_{q / p^{\uparrow}}\left(x_{\mathrm{B}}, \boldsymbol{k}_{T}\right)$ in their transverse momentum $\boldsymbol{k}_{T}[4,5]$

$$
f_{q / p^{\uparrow}}\left(x_{\mathrm{B}}, \boldsymbol{k}_{T}\right)=f_{1}^{q}\left(x_{\mathrm{B}}, k_{T}^{2}\right)-f_{1 T}^{\perp q}\left(x_{\mathrm{B}}, k_{T}^{2}\right) \frac{\left(\hat{\boldsymbol{P}} \times \boldsymbol{k}_{T}\right) \cdot \boldsymbol{S}}{M},
$$

where $\boldsymbol{S}$ is the spin of the target nucleon and $\hat{\boldsymbol{P}}$ is a unit vector opposite to the direction of the virtual photon momentum. The fact that such a term may be present in (3) is known as the Sivers effect and the function $f_{1 T}^{\perp q}\left(x_{\mathrm{B}}, k_{T}^{2}\right)$ is known as the Sivers function. The latter vanishes in a naive parton picture since $\left(\hat{\boldsymbol{P}} \times \boldsymbol{k}_{T}\right) \cdot \boldsymbol{S}$ is odd under naive time reversal (a property known as naive-T-odd), where one merely reverses the direction of all momenta and spins without interchanging the initial and final states. The momentum fraction $x$, which is equal to $x_{\mathrm{B}}$ in DIS experiments, represents the longitudinal momentum of the quark before it absorbs the virtual photon, as it is determined solely from the kinematic properties of the virtual photon and the target nucleon. In contradistinction, the transverse momentum $\boldsymbol{k}_{T}$ is defined in terms of the kinematics of the final state and hence it represents the asymptotic transverse momentum of the active quark after it has left the target and before it fragments into hadrons. Thus the Sivers function for semi-inclusive DIS includes the final state interaction between struck quark and target remnant, and time reversal invariance no longer requires that it vanishes. Indeed, as time reversal 
Figure 1. In SIDIS (a) the ejected (red) quark is attracted by the (anti-red) spectators. In contradistinction, in DY (b), before annihilating with the (red) active quark, the approaching (anti-red) antiquark is repelled by the (anti-red) spectators.

not only reverses the signs of all spins and momenta, but also transforms final state interactions (FSI) into initial state interactions (ISI), it has been shown that the Sivers function relevant for SIDIS and that relevant for Drell-Yan (DY) processes must have opposite signs [6],

$$
f_{1 T}^{\perp}\left(x_{\mathrm{B}}, k_{T}^{2}\right)_{S I D I S}=-f_{1 T}^{\perp}\left(x_{\mathrm{B}}, k_{T}^{2}\right)_{D Y}
$$

where the asymmetry in DY arises from the ISI between the incoming antiquark and the target. The experimental verification of this relation would provide a test of the current understanding of the Sivers effect within QCD. It is instructive to elucidate its physical origin in the context of a perturbative picture: for instance, when the virtual photon in a DIS process hits a red quark, the spectators must be collectively anti-red in order to form a colorneutral bound state, and thus attract the struck quark (Fig. 1). In DY, when an anti-red antiquark annihilates with a target quark, the target quark must be red in order to merge into a photon, which carries no color. Since the proton was colorless before the scattering, the spectators must be anti-red and thus repel the approaching antiquark.

The significant distortion of parton distributions in impact parameter space (2) provides a natural mechanism for a Sivers effect. In semi-inclusive DIS, when the virtual photon strikes a $u$ quark in a $\perp$ polarized proton, the $u$ quark distribution is enhanced on the left side of the target (for a proton with spin pointing up when viewed from the virtual photon perspective). Although in general the final state interaction (FSI) is very complicated, we expect it to be on average attractive thus translating a position space distortion to the left into a momentum space asymmetry to the right and vice versa (Fig. 2). Since this picture is very intuitive, a few words of caution are in order. First of all, such a reasoning is strictly valid only in mean field models for the FSI as well as in simple spectator models [7]. Furthermore, even in such mean field models there is no one-to-one correspondence between quark distributions in impact parameter space and unintegrated parton densities (e.g. Sivers function). 


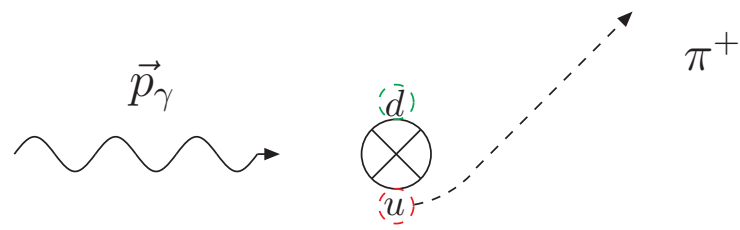

Figure 2. The transverse distortion of the parton cloud for a proton that is polarized into the plane, in combination with attractive FSI, gives rise to a Sivers effect for $u(d)$ quarks with a $\perp$ momentum that is on the average up (down).

While both are connected by a Wigner distribution [8], they are not Fourier transforms of each other. Nevertheless, since the primordial momentum distribution of the quarks (without FSI) must be symmetric we find a qualitative connection between the primordial position space asymmetry and the momentum space asymmetry (with FSI). Another issue concerns the $x$-dependence of the Sivers function. The $x$-dependence of the position space asymmetry is described by the GPD $E\left(x, 0,-\Delta_{\perp}^{2}\right)$. Therefore, within the above mechanism, the $x$ dependence of the Sivers function should be related to the $x$ dependence of $E\left(x, 0,-\Delta_{\perp}^{2}\right)$. However, the $x$ dependence of $E$ is not known yet and we only know the Pauli form factor $F_{2}=\int \mathrm{d} x E$. Nevertheless, if one makes the additional assumption that $E$ does not fluctuate as a function of $x$ then the contribution from each quark flavor $q$ to the anomalous magnetic moment $\kappa$ determines the sign of $E^{q}(x, 0,0)$ and hence of the Sivers function. Making these assumptions, as well as the very plausible assumption that the FSI is on average attractive, one finds that $f_{1 T}^{\perp \mu}<0$, while $f_{1 T}^{\perp d}>0$. Both signs have been confirmed by a flavor analysis based on pions produced in a SIDIS experiment by the Hermes collaboration [9].

\section{Charge Density in the Center of the Neutron}

As integrating the GPD $H^{q}$ over the momentum fraction $x$ of the active quark yields the Dirac form factor $F_{1}^{q}$, integrating Eq.(1) over $x$ also provides an interpretation of the Dirac form factor $F_{1}^{q}$ as the $2 \mathrm{~d}$ Fourier transform of the charge density (from quarks with flavor $q$ ) in impact parameter space

$$
\rho\left(\mathbf{b}_{\perp}\right)=\int \frac{\mathrm{d}^{2} \boldsymbol{\Delta}_{\perp}}{(2 \pi)^{2}} e^{i \mathbf{b}_{\perp} \cdot \boldsymbol{\Delta}_{\perp}} F_{1}^{q}\left(t=-\boldsymbol{\Delta}_{\perp}^{2}\right) .
$$

The main advantage of Eq. (5) compared to the Fourier transform of the Sachs form factors is that $\rho\left(\mathbf{b}_{\perp}\right)$ has a density interpretation $[2,10]$.

Application of Eq.(5) to $F_{1}$ for the neutron [11], yields a charge density $\rho\left(\mathbf{b}_{\perp}\right)$ that is negative not only at very large $\mathbf{b}_{\perp}$ but also near $\mathbf{b}_{\perp}=0$. The 
negative charge density at large distances $\mathbf{b}_{\perp}$ has the well known interpretation in terms of the pion cloud through the virtual process $n \rightarrow p \pi^{-}$, but the negative charge density near the origin appears to be mysterious. The key for intuitively understanding the negative charge density in the center of the neutron seems to be Orbital Angular Momentum (OAM).

With the benefit of hindsight, the first evidence for the presence of OAM in the nucleon wave function came from the existence of a large anomalous magnetic moment $\kappa_{p}=1.79$ and $\kappa_{n}=-1.91$. Indeed, in a relativistic theory, an anomalous magnetic moment necessarily implies the presence of wave function components with nonzero OAM (for a detailed discussion, see e.g. Refs. $[12,13])$. Further insight can be gained by performing a flavor decomposition of the anomalous magnetic moment. Neglecting the small contribution from strange and heavier quarks and making use of charge symmetry, one finds for the contribution from $u$ and $d$ quarks

$$
\kappa_{p}^{u}=\kappa_{n}^{d}=1.67 \quad \kappa_{p}^{d}=\kappa_{n}^{u}=-1.91
$$

respectively. Here charge factors have been taken out such that for example $\kappa_{p}=1.79=\frac{2}{3} \kappa_{p}^{u}-\frac{1}{3} \kappa_{p}^{d}$. For the purpose of this paper, we observe that not only are $\kappa_{N}^{q}$ large but that the magnitude of the contribution from the minority flavor ( $d$ in the proton and $u$ in the neutron) is even slightly larger than that of the corresponding majority flavor. Given that there are less down quarks in the proton, in combination with the fact that a nonzero anomalous magnetic moment requires wave function components with OAM, this result suggests that a $d$ quark in a proton has a significantly higher probability to be found with nonzero OAM than a $u$ quark.

The second piece of evidence comes from studies of the Sivers function $f_{1 T}^{\perp}$. A recent flavor analysis based on pions produced in SIDIS suggests a nonzero Sivers function for both $u$ and $d$ quarks with approximately equal magnitude and opposite sign $f_{u / p^{\uparrow}} \approx-f_{d / p^{\uparrow}}[9]$. Again we note that even though the proton contains more $u$ than $d$ quarks, the Sivers function for $d$ quarks is comparable in magnitude with those for $u$ quarks, again indicating that $d$ quark wave function components have a larger $p$ wave component.

Finally, we turn our attention to recent lattice calculations. Using the Ji relation $J_{q}=\frac{1}{2} \int \mathrm{d} x x\left[H_{q}(x, 0,0)+E_{q}(x, 0,0)\right]$ to determine the contribution $J_{q}$ of quark flavor $q$ to the nucleon spin from the GPDs $H_{q}$ and $E_{q}$ [14], and after subtracting the quark spin contribution, one finds [15]

$$
L_{u} \approx-L_{d} \approx 0.15
$$

i.e. about equal in magnitude and with opposite sign. While it is not entirely clear how to relate the OAM obtained through the Ji relation, to the OAM in 
light-cone wave function (the latter being relevant for the anomalous magnetic moment and the Sivers function) this result confirms our observation that the smaller number of $d$ quarks yields the same magnitude for the OAM, i.e. again a larger contribution from each $d$ quark (in the proton).

Despite the fact that there are less $d$ than $u$ quarks in the proton, they contribute with about the same magnitude to the anomalous magnetic moment, the Sivers function, and the quark OAM from the Ji-relation. These observations indicate that the wave function for $d$ quarks (in a proton) has a larger $p$-wave component than the one for $u$ quarks. Charge symmetry implies that in a neutron $u$ quarks have a larger $p$ wave components than $d$ quarks. Since $p$ wave function components are suppressed at the origin, this naturally suppresses $u$ quarks in a neutron for small $\mathbf{b}_{\perp}$ compared to an $S U(2)$ symmetric solution thus providing a qualitative explanation for the surprising result from Ref. [11].

\section{Tensor Correlations}

Another set of observables that are sensitive to spin-orbit correlations are Transverse Momentum dependent parton Distributions (TMDs). Projecting out quarks with transverse spin $\mathbf{s}$, the most general expression for the $\mathbf{k}_{T^{-}}^{2}$ dependence of parton distributions reads [16]

$$
\begin{aligned}
q\left(x, \mathbf{k}_{T}, \mathbf{s}, \mathbf{S}\right)=\frac{1}{2}[ & f_{1}+s^{i} S^{i} h_{1}+\frac{1}{M} S^{i} \varepsilon^{i j} k^{j} f_{1 T}^{\perp}+\frac{1}{M} s^{i} \varepsilon^{i j} k^{j} h_{1}^{\perp} \\
& \left.+\frac{1}{M} \Lambda s^{i} k^{i} h_{1 L}^{\perp}+\frac{1}{2 M^{2}} s_{i} S_{j}\left(2 k^{i} k^{j}-\mathbf{k}_{T}^{2} \delta^{i j}\right) h_{1 T}^{\perp}\right],
\end{aligned}
$$

where $\Lambda$ is the longitudinal nucleon polarization and $\mathbf{S}$ its transverse spin. Two more terms appear when one also considers longitudinally polarized quarks.

In the following, we will focus on the chirally odd tensor correlation $h_{1 T}^{\perp}$, which contributes to matrix elements with a double spin asymmetry in orthogonal transverse directions. In a helicity basis, this implies that it contributes to matrix elements where both quark and nucleon helicities flip — but in opposite directions - resulting in a helicity mismatch by two units. A specific example is the transition from a nucleon state with $S_{z}=+\frac{1}{2}$ to $S_{z}=-\frac{1}{2}$ while the spin of the active quark flips from $s_{z}=-\frac{1}{2}$ to $s_{z}=+\frac{1}{2}$. The active quark thus has to absorb $L_{z}=-2$. The fact that $L_{z}$ changes by two units requires either the presence of wave function components with $L_{z}= \pm 2$ ( $s$ - $d$ interference), or matrix elements that are quadratic in the $p$ wave component. In either case, applying the power-counting techniques from Ref. [17], this implies that $h_{1 T}^{\perp} \stackrel{x \rightarrow 1}{\longrightarrow}(1-x)^{5}$. 
We will focus on contributions quadratic in the $p$ wave component and neglect $s$ - $d$ interference. Consider a nucleon that is polarized in the $+\hat{x}$ direction. When the active quark has $l_{x}= \pm 1$, its distribution is enhanced in the $\hat{y}-\hat{z}$ plane. When viewed from the $\hat{z}$ direction, the distribution is thus enhanced along the $\hat{y}$ axis, but suppressed along the $\hat{x}$ axis (imagine a bagel in the $\hat{y}-\hat{z}$ plane viewed from the side). On the other hand, for $l_{x}=0$, the quark distribution is enhanced along the $\hat{x}$ axis (peanut aligned along with the $\hat{x}$ axis). As this deformation is described by $h_{1 T}^{\perp}$, this TMD thus appears the ideal tool to decide whether (and in which spin configuration) the polarized quark density looks more like a bagel or a peanut, or perhaps even a pretzel [18].

In order to better understand the specific implications for the nucleon's angular momentum structure, let us consider for example the case $h_{1 T}^{\perp}<$ 0 . According to the above discussion, this case corresponds to $l_{x}=0$ when quark and nucleon transversity are anti-parallel, and $l_{x}= \pm 1$ when they are parallel. In quark models the tensor correlation $h_{1 T}^{\perp}$ usually arises solely from the lower component (standard representation for Dirac matrices) and for the lower component transverse spin and transversity have opposite signs. Thus the case $h_{1 T}^{\perp}<0$ corresponds to $l_{x}=0$ when quark and nucleon transverse spin are parallel, and $l_{x}= \pm 1$ when they are anti-parallel. In a bag model or potential model, this type of correlation arises naturally when $j_{q}$ is in the same direction as the nucleon spin, as the $p$ wave component arises from the lower component of the quark wave function, and is largest when $j_{q}$ and $s_{q}$ are antiparallel with $l_{q}$ parallel to $j_{q}$. In most models, one would thus expect $h_{1 T}^{\perp u}<0$. The case $h_{1 T}^{\perp}>0$ corresponds to $l_{x}= \pm 1$ when quark and nucleon transversity are parallel (transverse spins anti-parallel), and $l_{x}=0$ when they are antiparallel (transverse spins parallel). This naturally arises in quark states with $l_{q}$ and $s_{q}$ coupled to a net $j_{q}$ that is oriented opposite to the nucleon spin. We thus expect $h_{1 T}^{\perp d}>0$ with smaller absolute magnitude than $h_{1 T}^{\perp u}$.

In contradistinction to the Sivers and Boer-Mulders functions, $h_{1 T}^{\perp}$ is (naive) T-even and does not require the presence of nontrivial ISI/FSI phases. Nevertheless, measurements of $h_{1 T}^{\perp}$ in SIDIS or DY experiments may still be affected by ISI/FSI. While such effects may be small compared to the intrinsic $h_{1 T}^{\perp}$, it is not a priori clear how to separate ISI/FSI from intrinsic effects.

In Ref. [19] it has been shown that a tensor correlation analogous to the one described by $h_{1 T}^{\perp}$ in momentum space is described by the Fourier transform of the GPD $\tilde{H}_{T}^{\prime \prime}$ in impact parameter space. There the issue of ISI/FSI does not arise, as the GPDs are defined through matrix elements of local operators and directly probe the intrinsic quark densities. While it is not possible to directly map a density in position space onto a density in momentum, (single particle) $p$-orbits have the same angular distribution in momentum space as they have in 
position space. Therefore, a bagel in momentum space corresponds to a bagel in position space as well and one would thus expect that $h_{1 T}^{\perp}$ has the same sign as $\tilde{H}_{T}^{\prime \prime}$. Generalizing the approach from Ref. 20, more concrete relations between tensor correlation in momentum and impact-parameter space have been derived in Ref. 21 for the specific case of diquark models.

\section{References}

1. M.Burkardt, Phys. Rev. D 62, 071503 (2000), Erratum-ibid. D 66, 119903 (2002).

2. D. Soper. Phys. Rev. D 5, 1956 (1972).

3. M. Burkardt, Phys. Rev. D 66, 114005 (2002); Phys. Rev. D 69, 057501 (2004).

4. D.W. Sivers, Phys. Rev. D 43, 261 (1991).

5. A. Bacchetta et al., Phys. Rev. D 70, 117504 (2004).

6. J.C. Collins, Phys. Lett. B 536, 43 (2002).

7. S.J. Brodsky, D.S. Hwang, and I. Schmidt, Nucl. Phys. B 642, 344 (2002); M. Burkardt and D.S. Hwang, Phys. Rev. D69, 074032 (2004); L.P. Gamberg et al., Phys. Rev. D 67, 071504 (2003); D. Boer, S.J. Brodsky, and D.S. Hwang, Phys. Rev. D 67, 054003 (2003); A. Bacchetta et al., Phys. Lett B578, 109 (2004); M. Radici et al., hep-ph/0708.0232; L.P. Gamberg et al. hep-ph/0708.0324; D. Boer et al., Phys. Rev. D 67, 054003 (2003); L.P. Gamberg et al., Phys. Rev. D 67 (2003) 071504.

8. A.V. Belitsky, X. Ji, and F. Yuan, Phys. Rev. D 69, 074014 (2004).

9. A. Airapetian et al. (Hermes collaboration), Phys. Rev. Lett. 94, 012002 (2005).

10. M. Burkardt, proc. Workshop on Lepton Scattering, Hadrons and QCD, Adelaide, Australia, March 2001, hep-ph/0105324; P.V. Pobylitsa, Phys. Rev. D 66, 094002 (2002).

11. G.A. Miller, arXiv:0705.2409

12. M. Burkardt and G. Schnell, Phys. Rev. D 74, 013002 (2006).

13. S.J. Brodsky and S. Gardner, Phys. Lett. B 643, 22 (2006).

14. X. Ji, Phys. Rev. Lett. 78, 610 (1997).

15. M. Göckeler et al. (QCDSF collaboration), Phys. Rev. Lett. 98, 222001 (2007);

Ph. Hägler et al. (LHPC collaboration), hep-lat/0705.4295.

16. A. Bacchetta et al., Phys. Rev. Lett. 85, 712 (2000).

17. H. Avakian et al., hep-ph/0705.1553

18. G.A. Miller, Phys. Rev. C 68, 022201 (2003); G.A. Miller, arXiv:0708.2297

19. M. Diehl and P.Hägler, Eur. Phys. J. C44, 87 (2005).

20. Z. Lu and I. Schmidt, Phys. Rev. D 75, 073008 (2007).

21. S. Meissner, A. Metz, and K. Goeke, Phys. Rev. D 76, 034 (2007). 


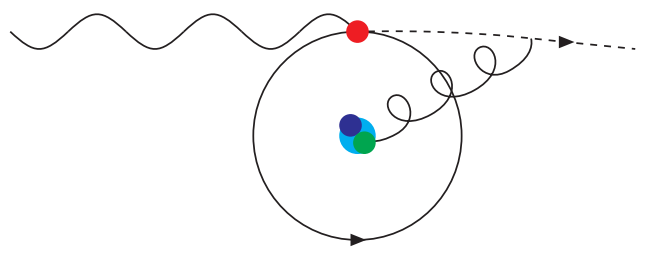

a)

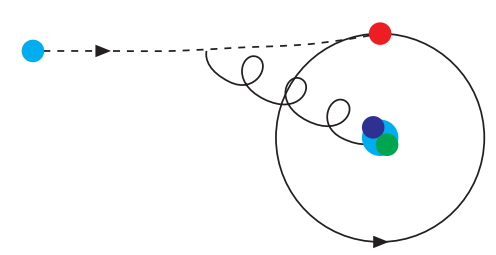

b) 